Pacific

Journal of

Mathematics

VASSILIEV INVARIANTS OF KNOTS IN A SPATIAL

GRAPH

Yoshiyuki OHyama AND Kouki Taniyama

Volume $200 \quad$ No. 1 



\title{
VASSILIEV INVARIANTS OF KNOTS IN A SPATIAL GRAPH
}

\author{
Yoshiyuki OHyama and Kouki Taniyama \\ Dedicated to Professor Kazuaki Kobayashi for his 60th birthday.
}

\begin{abstract}
We show that the Vassiliev invariants of the knots contained in an embedding of a graph $G$ into $R^{3}$ satisify certain equations that are independent of the choice of the embedding of $G$. By a similar observation we define certain edge-homotopy invariants and vertex-homotopy invariants of spatial graphs based on the Vassiliev invariants of the knots contained in a spatial graph. A graph $G$ is called adaptable if, given a knot type for each cycle of $G$, there is an embedding of $G$ into $R^{3}$ that realizes all of these knot types. As an application we show that a certain planar graph is not adaptable.
\end{abstract}

\section{Introduction.}

Throughout this paper we work in the piecewise linear category. Let $G$ be a finite loopless graph. Let $\operatorname{SE}(G)$ be the set of all embeddings of $G$ into the three-dimensional Euclidean space $R^{3}$. An element of $\operatorname{SE}(G)$ is called a spatial embedding of $G$. The image of a spatial embedding of $G$ is also called a spatial embedding of $G$ so long as no confusion occurs. A spatial embedding of a graph is called a spatial graph. The study of spatial graphs up to ambient isotopy is a branch of knot theory. The knots and links contained in a spatial graph are primitive invariants of the spatial graph. The following interesting theorem on knots and links in a spatial graph is shown in $[\mathbf{3}]$. Let $K_{n}$ be the complete graph on $n$ vertices.

Theorem $1.1([3])$. (1) Every spatial embedding of $K_{6}$ contains a nontrivial link.

(2) Every spatial embedding of $K_{7}$ contains a nontrivial knot.

In fact the following theorem is shown in $[\mathbf{3}]$.

Theorem $1.2([3])$. (1) For any element $f$ of $\operatorname{SE}\left(K_{6}\right)$, the sum of the linking numbers of all two-component links contained in $f\left(K_{6}\right)$ is odd.

(2) For any element $f$ of $\mathrm{SE}\left(K_{7}\right)$, the sum of the Arf invariants of all knots in $f\left(K_{7}\right)$ containing all vertices of $f\left(K_{7}\right)$ is odd. 
Since the linking number of a trivial two-component link is zero and the Arf invariant of a trivial knot is zero, Theorem 1.1 is an immediate corollary of Theorem 1.2. The above theorems imply that the knots and links in a spatial graph are mutually dependent in general. We refer the reader to $[\mathbf{1 6}],[\mathbf{7}],[\mathbf{1 4}]$ and $[\mathbf{1 5}]$ for related results and $[\mathbf{2 1}]$ for a higher dimensional analogue.

The main purpose of this paper is to give a generalization of Theorem 1.2. We show that the Vassiliev type invariants of order at most $n$ of the knots contained in a spatial embedding of $G$ satisfy certain equations that are independent of the choice of the embedding of $G$ if the graph $G$ is sufficiently large in comparison with $n$. We remark here that the linking number and the Arf invariant are the Vassiliev type invariants of order 1 and 2 respectively.

We refer the reader to $[\mathbf{2 3}],[\mathbf{2}],[\mathbf{1}],[\mathbf{4}],[\mathbf{1 7}],[\mathbf{8}],[\mathbf{1 2}],[\mathbf{1 3}]$ etc. for Vassiliev type invariants.

A graph is called planar if it is embeddable into a plane. A plane graph is a spatial graph that is ambient isotopic to a spatial graph on a plane in $R^{3}$. As an application we show in Corollary 3.2 that the knot types in a spatial embedding of the graph in Figure 1.1 satisfy a nontrivial condition. In fact we have, for example, that there is no embedding of the graph in Figure 1.1 that contains just one trefoil knot containing all of the vertices of the graph, and contains no other nontrivial knots. Note that the graph in Figure 1.1 is the first planar graph which is known to have such a property.

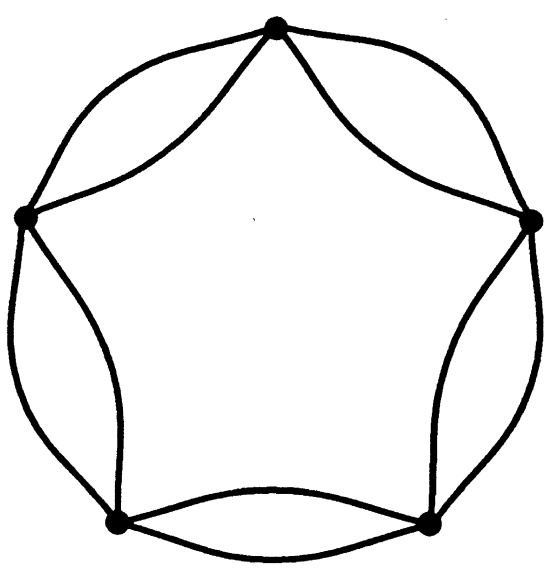

Figure 1.1.

Then we define certain edge-homotopy invariants and vertex-homotopy invariants of spatial graphs based on the Vassiliev type invariants of the knots contained in a spatial graph. Here edge-homotopy allows crossing changes of an edge with itself and vertex-homotopy allows crossing changes of two adjacent edges. Then we show in Example 3.6 that none of the graphs in Figure 1.2 is edge-homotopic to a plane graph. 

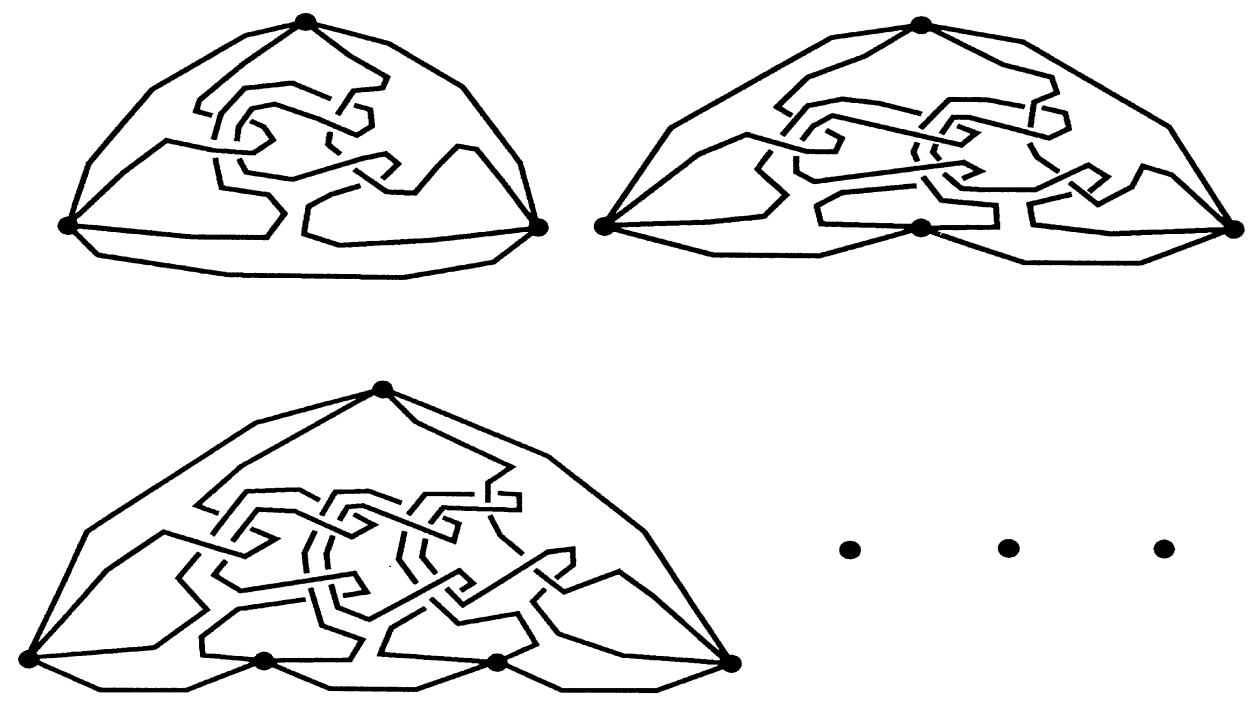

Figure 1.2.

\section{General results.}

Let $\mathcal{R}$ be a commutative ring with unit 1 . Let $v: \mathrm{SE}(G) \rightarrow \mathcal{R}$ be an ambient isotopy invariant. Namely, $v$ is a map from $\operatorname{SE}(G)$ to $\mathcal{R}$ such that $v(f)=v(g)$ for any ambient isotopic embeddings $f$ and $g$. Let $\mathrm{SE}_{i}(G)$ be the set of all $i$-singular embeddings of $G$ into $R^{3}$ where an $i$-singular embedding is a continuous map whose multiple points are exactly $i$ double points of edges spanning small flat planes. Such a double point is called a crossing vertex. Then, under a given edge orientation of $G, v$ is uniquely extended to an ambient isotopy invariant $v_{i}: \mathrm{SE}_{i}(G) \rightarrow \mathcal{R}$ by the recursive formula

$$
v_{i}\left(f_{0}\right)=v_{i-1}\left(f_{+}\right)-v_{i-1}\left(f_{-}\right)
$$

where $f_{0}, f_{+}$and $f_{-}$are related as illustrated in Figure 2.1.

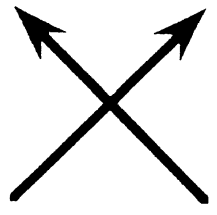

$f_{0}$

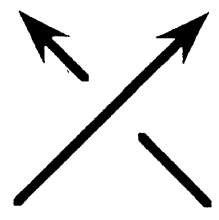

$f_{+}$

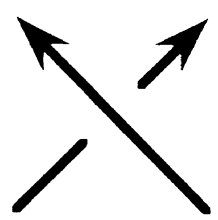

$f_{-}$

Figure 2.1.

Here we only consider ambient isotopies that preserve a small flat plane at each crossing vertex. We say that $f_{+}$(resp. $f_{-}$) is a positive (resp. negative) 
resolution of $f_{0}$ at the crossing vertex illustrated in Figure 2.1. Let $n$ be a natural number. We say that $v$ is a Vassiliev type invariant of order at most $n$ if $v_{n+1}: \mathrm{SE}_{n+1}(G) \rightarrow \mathcal{R}$ is a zero map. (Then we have that $v_{m}$ is a zero map for $m \geq n+1$.) It is easy to check that the definition of Vassiliev type invariant of order at most $n$ is independent of the choice of the edge orientations.

Now we fix a ring $\mathcal{R}$ and a natural number $n$. Let $\Omega(G)$ be the set of all subgraphs of $G$. Let $\Gamma$ be a subset of $\Omega(G)$. Suppose that for each $\gamma \in \Gamma$, a Vassiliev type invariant of order at most $n v_{\gamma}: \operatorname{SE}(\gamma) \rightarrow \mathcal{R}$ is given. (Possibly $v_{\gamma}$ is a zero map.) Let $\omega: \Gamma \rightarrow \mathcal{R}$ be a map. Then we define an ambient isotopy invariant $v=v\left(\left\{v_{\gamma}\right\}, \omega\right): \mathrm{SE}(G) \rightarrow \mathcal{R}$ by

$$
v(f)=\sum_{\gamma \in \Gamma} \omega(\gamma) v_{\gamma}\left(\left.f\right|_{\gamma}\right)
$$

Note that when $\Gamma=\{G\}$ and $\omega(G)=1 v$ is just a Vassiliev type invariant of $G$ of order at most $n$. We include this case in the following. In general we have the following proposition.

Proposition 2.1. $v$ is a Vassiliev type invariant of order at most $n$.

Proof. Let $f$ be an element of $\mathrm{SE}_{n+1}(G)$ and $c_{1}, \cdots, c_{n+1}$ the crossing vertices of $f$. Let $P$ be a subset of $\{1, \cdots, n+1\}$. By $f_{P}$ we denote an element of $\operatorname{SE}(G)$ that is obtained from $f$ by resolving $c_{i}$ positively if $i$ is contained in $P$ and negatively if $i$ is not contained in $P$. Then we have

$$
\begin{aligned}
v_{n+1}(f) & =\sum_{P \subset\{1, \cdots, n+1\}}(-1)^{n+1-|P|} v\left(f_{P}\right) \\
& =\sum_{P \subset\{1, \cdots, n+1\}}(-1)^{n+1-|P|}\left(\sum_{\gamma \in \Gamma} \omega(\gamma) v_{\gamma}\left(f_{P} \mid \gamma\right)\right) \\
& =\sum_{\gamma \in \Gamma} \omega(\gamma)\left(\sum_{P \subset\{1, \cdots, n+1\}}(-1)^{n+1-|P|} v_{\gamma}\left(f_{P} \mid \gamma\right)\right) .
\end{aligned}
$$

Therefore it is sufficient to show that

$$
\sum_{P \subset\{1, \cdots, n+1\}}(-1)^{n+1-|P|} v_{\gamma}\left(\left.f_{P}\right|_{\gamma}\right)=0
$$

for each $\gamma \in \Gamma \subset \Omega(G)$.

First suppose that some $c_{i}$ is not a crossing vertex of $\left.f\right|_{\gamma}$. Then for each $P \subset\{1, \cdots, n+1\}$ with $i \in P,\left.f_{P}\right|_{\gamma}$ and $\left.f_{P-\{i\}}\right|_{\gamma}$ are ambient isotopic. 
Therefore

$$
\begin{aligned}
& \sum_{P \subset\{1, \cdots, n+1\}}(-1)^{n+1-|P|} v_{\gamma}\left(\left.f_{P}\right|_{\gamma}\right) \\
= & \sum_{P \subset\{1, \cdots, n+1\}, P \ni i}\left((-1)^{n+1-|P|} v\left(\left.f_{P}\right|_{\gamma}\right)+(-1)^{n+1-(|P|-1)} v\left(f_{P-\{i\}} \mid \gamma\right)\right)=0 .
\end{aligned}
$$

Next suppose that every $c_{i}$ is a crossing vertex of $\left.f\right|_{\gamma}$. Then we have that $\left.f\right|_{\gamma} \in \mathrm{SE}_{n+1}(\gamma)$ and

$$
\sum_{P \subset\{1, \cdots, n+1\}}(-1)^{n+1-|P|} v_{\gamma}\left(\left.f_{P}\right|_{\gamma}\right)=\left(v_{\gamma}\right)_{n+1}\left(\left.f\right|_{\gamma}\right)=0 .
$$

An $i$-configuration on $G$ is a pairing of $2 i$ points on the edges of $G$. A realization of an $i$-configuration is an element of $\mathrm{SE}_{i}(G)$ whose crossing vertices correspond to the pairing. Let $e_{1}$ and $e_{2}$ be edges of $G$ (possibly $e_{1}=e_{2}$ ). We say that $f$ and $g$ in $\mathrm{SE}(G)$ are $\left(e_{1}, e_{2}\right)$-homotopic if $g$ is obtained from $f$ by a series of crossing changes between $e_{1}$ and $e_{2}$ and ambient isotopy. Here a crossing change between $e_{1}$ and $e_{2}$ is a change of a crossing whose over-path belongs to an image of $e_{1}$ and under-path belongs to an image of $e_{2}$.

Theorem S. The following conditions are mutually equivalent.

(1) $v(f)=v(g)$ for any $\left(e_{1}, e_{2}\right)$-homotopic embeddings $f$ and $g$ in $\mathrm{SE}(G)$,

(2) $v_{i}(f)=0$ for any $f$ in $\mathrm{SE}_{i}(G)$ with $1 \leq i \leq n$ that has at least one crossing vertex of $e_{1}$ and $e_{2}$,

(3) for any $i$-configuration $C$ on $G$ with $1 \leq i \leq n$ that has at least one pair of points one on $e_{1}$ and the other on $e_{2}$, there is a realization $f_{C} \in \operatorname{SE}_{i}(G)$ of $C$ such that $v_{i}\left(f_{C}\right)=0$.

Proof. (1) $\rightarrow(2)$ It is sufficient to show that $v_{1}(f)=0$ for any 1-singular embedding $f \in \mathrm{SE}_{1}(G)$ that has just one crossing vertex of $e_{1}$ and $e_{2}$. The two resolusions $f_{+}$and $f_{-}$of $f$ are $\left(e_{1}, e_{2}\right)$-homotopic embeddings. Therefore $v\left(f_{+}\right)=v\left(f_{-}\right)$. Thus $v_{1}(f)=v\left(f_{+}\right)-v\left(f_{-}\right)=0$.

$(2) \rightarrow(1)$ It is sufficient to show the case that $g$ is obtained from $f$ by a crossing change of $e_{1}$ and $e_{2}$. Let $h \in \mathrm{SE}_{1}(G)$ be the 1-singular embedding that corresponds to the crossing change between $f$ and $g$. Then we have $v(f)-v(g)=v_{1}(h)=0$.

$(2) \rightarrow(3)$ It is clear.

$(3) \rightarrow(2)$ First we show that $v_{n}(f)=0$ for any $f$ in $\operatorname{SE}_{n}(G)$ that has at least one crossing vertex of $e_{1}$ and $e_{2}$. Let $C$ be the $n$-configuration on $G$ that corresponds to $f$. Let $f_{C}$ be the realization of $C$ with $v_{n}\left(f_{C}\right)=0$. We note that $f$ and $f_{C}$ are transformed into each other by a sequence of crossing changes and ambient isotopy. Since $v_{n+1}: \mathrm{SE}_{n+1}(G) \rightarrow \mathcal{R}$ is the 
zero map (Proposition 2.1) crossing changes do not change the value of $v_{n}$. Therefore we have $v_{n}(f)=v_{n}\left(f_{C}\right)=0$.

Next suppose inductively on $i$ that $v_{i}(f)=0$ for any $f$ in $\operatorname{SE}_{i}(G)$ that has at least one crossing vertex of $e_{1}$ and $e_{2}$. Then similarly we have that $v_{i-1}(f)=0$ for any $f$ in $\mathrm{SE}_{i-1}(G)$ that has at least one crossing vertex of $e_{1}$ and $e_{2}$. This completes the proof.

As follows we can actually check whether or not these conditions hold. For each $i$-configuration $C$ on $G$ with $1 \leq i \leq n$ that has at least one pair of points one on $e_{1}$ and the other on $e_{2}$, choose any realization $f_{C} \in \mathrm{SE}_{i}(G)$ of $C$. If $v_{i}\left(f_{C}\right) \neq 0$ for some configuration $C$ then we have that the condition (2) does not hold. If $v_{i}\left(f_{C}\right)=0$ for each configuration $C$ then we have that the condition (3) holds.

We say that $f$ and $g$ in $\mathrm{SE}(G)$ are

(1) edge-homotopic if $g$ is obtained from $f$ by a series of "self-crossing changes" and ambient isotopy. Here a self-crossing change is a change of a crossing whose over-path and under-path belong to an edge of $G$. Namely, edge-homotopy is the equivalence relation generated by $(e, e)$-homotopies for all edges $e$,

(2) vertex-homotopic if $g$ is obtained from $f$ by a series of "crossing changes between adjacent edges" and ambient isotopy. Here a crossing change between adjacent edges is a change of a crossing whose over-path and under-path belong to two edges that have a common vertex. Namely, vertex-homotopy is the equivalence relation generated by $\left(e_{1}, e_{2}\right)$-homotopies for all pair of adjecent edges $e_{1}, e_{2}$.

We note that edge-homotopy and vertex-homotopy are equivalence relations on spatial graphs introduced in [18] as generalizations of Milnor's link homotopy $[\mathbf{9}]$. We remark that edge-homotopy implies vertex-homotopy since $G$ is loopless [18].

The following three parallel theorems are immediate consequences of Theorem S.

Theorem A. The following conditions are mutually equivalent.

(1) $v(f)=v(g)$ for any $f$ and $g$ in $\operatorname{SE}(G)$,

(2) $v_{i}(f)=0$ for any $f$ in $\mathrm{SE}_{i}(G)$ with $1 \leq i \leq n$,

(3) for any $i$-configuration $C$ on $G$ with $1 \leq i \leq n$, there is a realization $f_{C} \in \mathrm{SE}_{i}(G)$ of $C$ such that $v_{i}\left(f_{C}\right)=0$.

Theorem B. The following conditions are mutually equivalent.

(1) $v(f)=v(g)$ for any edge-homotopic embeddings $f$ and $g$ in $\mathrm{SE}(G)$,

(2) $v_{i}(f)=0$ for any $f$ in $\operatorname{SE}_{i}(G)$ with $1 \leq i \leq n$ that has at least one "self-crossing vertex",

(3) for any $i$-configuration $C$ on $G$ with $1 \leq i \leq n$ that has at least one pair of points on an edge, there is a realization $f_{C} \in \mathrm{SE}_{i}(G)$ of $C$ such that $v_{i}\left(f_{C}\right)=0$. 
Theorem C. The following conditions are mutually equivalent.

(1) $v(f)=v(g)$ for any vertex-homotopic embeddings $f$ and $g$ in $\operatorname{SE}(G)$,

(2) $v_{i}(f)=0$ for any $f$ in $\mathrm{SE}_{i}(G)$ with $1 \leq i \leq n$ that has at least one "crossing vertex of adjacent edges",

(3) for any $i$-configuration $C$ on $G$ with $1 \leq i \leq n$ that has at least one pair of points on two edges that have a common vertex, there is a realization $f_{C} \in \mathrm{SE}_{i}(G)$ of $C$ such that $v_{i}\left(f_{C}\right)=0$.

Here a self-crossing vertex is a crossing vertex of a single edge and a crossing vertex of adjacent edges is a crossing vertex of two edges that have a common vertex. We note that we can actually check whether or not the conditions in each of Theorem A, Theorem B and Theorem C hold as before. The proofs of Theorem A, Theorem B and Theorem C easilly come from Theorem S and we omit them.

Suppose that for each $\gamma \in \Gamma \subset \Omega(G), \phi_{\gamma} \in \operatorname{SE}(\gamma)$ is given. Then we say that a set of spatial embeddings $\left\{\phi_{\gamma} \in \operatorname{SE}(\gamma) \mid \gamma \in \Gamma\right\}$ is realizable up to ambient isotopy if there is an element $f$ of $\operatorname{SE}(G)$ such that the restriction map $\left.f\right|_{\gamma}$ is ambient isotopic to $\phi_{\gamma}$ for all $\gamma \in \Gamma$. As an immediate corollary of Theorem A we have the following theorem.

Theorem 2.2. Suppose that the conditions of Theorem A hold. Let $f$ be an element of $\operatorname{SE}(G)$. Then a set of spatial embeddings $\left\{\phi_{\gamma} \in \operatorname{SE}(\gamma) \mid \gamma \in\right.$ $\Gamma\}$ is realizable up to ambient isotopy only if $\sum_{\gamma \in \Gamma} \omega(\gamma) v_{\gamma}\left(\phi_{\gamma}\right)=v(f)=$ $\sum_{\gamma \in \Gamma} \omega(\gamma) v_{\gamma}\left(\left.f\right|_{\gamma}\right)$.

Remark. (1) Since the mod 2 linking number and the Arf invariant are order 1 and 2 Vassiliev type invariants respectively, Theorem 1.2 serves examples of Theorem 2.2 where the ring $\mathcal{R}=Z / 2 Z$. In fact it is not hard to check that the condition (3) of Theorem A holds for $v$ the sum of the linking numbers of the links in a spatial embedding of $K_{6}$, and for $v$ the sum of the Arf invariants of the knots in a spatial embedding of $K_{7}$ each of which contains all of the vertices of $K_{7}$. The spatial embedding of $K_{6}$ illustrated in Figure 2.2 contains just one nontrivial link as in Figure 2.2 and the spatial embedding of $K_{7}$ illustrated in Figure 2.3 contains just one nontrivial knot as in Figure 2.3, cf. [14]. Thus we have Theorem 1.2.

(2) Since the second coefficient of the Conway polynomial of a knot is an order 2 Vassiliev type invariant, the edge-homotopy (resp. vertex-homotopy) invariants defined in $[\mathbf{1 9}]$ are examples of Theorem B (resp. Theorem C). 

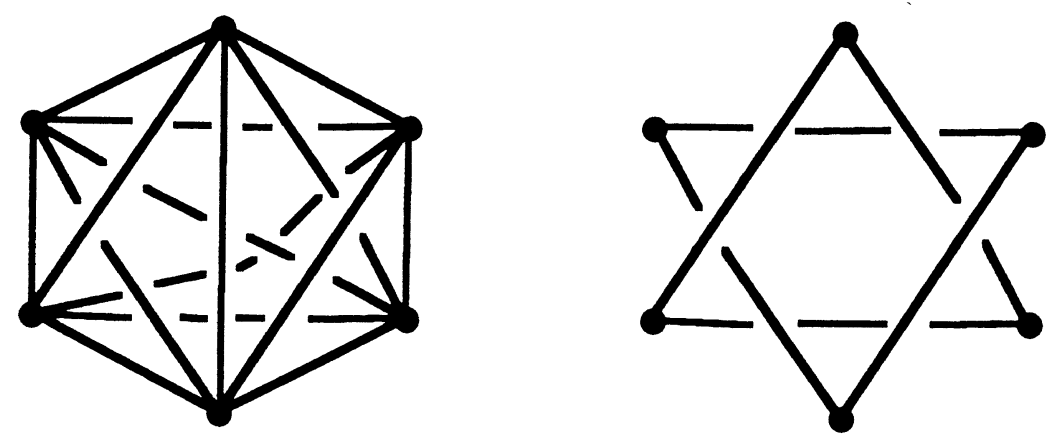

Figure 2.2.
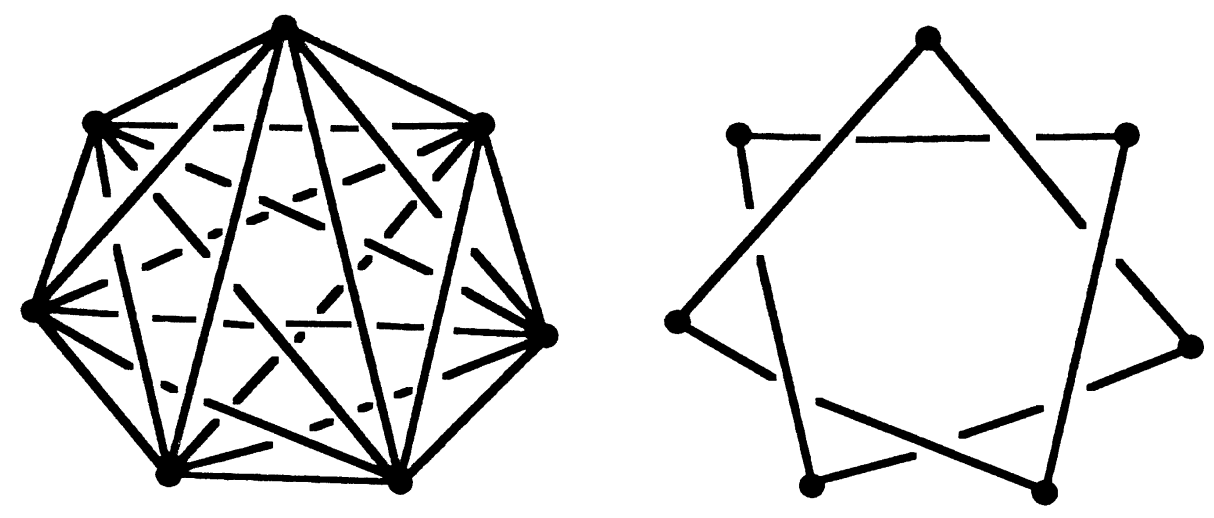

Figure 2.3.

We sometimes restrict our attension to certain class of embeddings of a graph $G$ and find some relations among the spatial embeddings of the subgraphs of $G$ that may not hold in an arbitrary embedding of $G$.

Theorem D. Let $\mathrm{T}_{i}(G)$ be a subset of $\mathrm{SE}_{i}(G)$ for each $i$ with $1 \leq i \leq n$ and $\mathrm{T}_{n+1}(G)=\mathrm{SE}_{n+1}(G)$. Suppose that for any $f \in \mathrm{T}_{i}(G)$ with $1 \leq i \leq n$ there is an element $g \in \mathrm{T}_{i}(G)$ with $v_{i}(g)=0$ and a sequence of crossing changes from $f$ to $g$ such that the corresponding $(i+1)$-singular embeddings of $G$ are contained in $\mathrm{T}_{i+1}(G)$. Then $v_{i}(h)=0$ for any $h \in \mathrm{T}_{i}(G)$ with $1 \leq i \leq n$.

Proof. First suppose that $h \in \mathrm{T}_{n}(G)$. Then by the assumption there is $u \in \mathrm{T}_{n}(G)$ with $v_{n}(u)=0$ and a sequence of crossing changes from $h$ to $u$ such that the corresponding $(n+1)$-singular embeddings are contained in $\mathrm{T}_{n+1}(G)=\mathrm{SE}_{n+1}(G)$. By Proposition 2.1 we have that $v_{n+1}$ is the zero map. Therefore we have $v_{n}(h)-v_{n}(u)=0$ and $v_{n}(h)=0$. Next suppose inductively that $v_{i+1}(h)=0$ for any $h \in \mathrm{T}_{i+1}(G)$. Let $h \in \mathrm{T}_{i}(G)$. Then 
there is $u \in \mathrm{T}_{i}(G)$ with $v(u)=0$ and a sequence of crossing changes from $h$ to $u$ whose corresponding $(i+1)$-singular embeddings are contained in $\mathrm{T}_{i+1}(G)$. Then we have $v(h)-v(u)=0$ and $v(h)=0$.

\section{Graphs with multiple edges.}

Let $e_{1}, \cdots, e_{m}$ be some edges of $G$. Let $G\left(e_{1}, \cdots, e_{m}\right)$ be a graph obtained by adding edges $d_{1}, \cdots, d_{m}$ to $G$ so that $e_{i}$ and $d_{i}$ form a pair of multiple edges for each $i$. Let $\Lambda$ be a subset of $\{1, \cdots, m\}$. Let $G_{\Lambda}$ be a subgraph of $G\left(e_{1}, \cdots, e_{m}\right)$ obtained from $G$ by replacing $e_{i}$ to $d_{i}$ for each $i \in \Lambda$. Then $G_{\Lambda}$ is canonically isomorphic to $G$. Let $\varphi_{\Lambda}: G \rightarrow G_{\Lambda}$ be a canonical homeomorphism. Let $\Gamma=\Gamma\left(e_{1}, \cdots, e_{m}\right)=\left\{G_{\Lambda} \mid \Lambda \subset\{1, \cdots, m\}\right\}$. We remark here that $G=G_{\emptyset}$ and the number of elements $|\Gamma|=2^{m}$. Let $v_{G}: \mathrm{SE}(G) \rightarrow \mathcal{R}$ be a Vassiliev type invariant of order at most $n$. Let $v_{G_{\Lambda}}: \operatorname{SE}\left(G_{\Lambda}\right) \rightarrow \mathcal{R}$ be the Vassiliev type invariant of order at most $n$ defined by $v_{G_{\Lambda}}(f)=v_{G}\left(f \circ \varphi_{\Lambda}\right)$. Let $\omega: \Gamma \rightarrow \mathcal{R}$ be a map defined by $\omega\left(G_{\Lambda}\right)=(-1)^{|\Lambda|}$. Then we have the following theorem from Theorem A, Theorem B and Theorem C.

Theorem 3.1. Let $v=v\left(\left\{v_{G_{\Lambda}}\right\}, \omega\right): \operatorname{SE}\left(G\left(e_{1}, \cdots, e_{m}\right)\right) \rightarrow \mathcal{R}$ be a map defined as above.

(1) If $n$ is less than $m / 2$ then $v$ is the zero map.

(2) If $n$ is less than $(m+2) / 2$ then $v$ is an edge-homotopy invariant.

(3) If $n$ is less than $(m+1) / 2$ then $v$ is a vertex-homotopy invariant.

Let $e$ and $d$ be a pair of multiple edges of $G$ and $f \in \operatorname{SE}_{i}(G)$. We say that $e$ and $d$ are parallel in $f$ if $f(e \cup d)$ contains no crossing vertices of $f$ and bounds a disk in $R^{3}$ whose interior is disjoint from $f(G)$.

Proof of Theorem 3.1 (1). Suppose that some edges $e_{l}$ and $d_{l}$ are parallel in $f \in \mathrm{SE}_{i}\left(G\left(e_{1}, \cdots, e_{m}\right)\right)$. Let $g \in \mathrm{SE}\left(G\left(e_{1}, \cdots, e_{m}\right)\right)$ be any one of $2^{i}$ total resolutions of $f$. Then we have that $e_{l}$ and $d_{l}$ are parallel in $g$. Then

$$
\begin{aligned}
& v_{i}(g) \\
& =\sum_{\Lambda \subset\{1, \cdots, m\}}(-1)^{|\Lambda|} v_{G_{\Lambda}}\left(\left.g\right|_{G_{\Lambda}}\right) \\
& =\sum_{\Lambda \subset\{1, \cdots, m\}}(-1)^{|\Lambda|} v_{G}\left(\left.g\right|_{G_{\Lambda}} \circ \varphi_{\Lambda}\right) \\
& =\sum_{\Lambda \subset\{1, \cdots, m\}, \Lambda \ni l}\left((-1)^{|\Lambda|} v_{G}\left(\left.g\right|_{G_{\Lambda}} \circ \varphi_{\Lambda}\right)+(-1)^{\left.|\Lambda-\{l\}|_{G}\left(\left.g\right|_{G_{\Lambda-\{l\}}} \circ \varphi_{\Lambda-\{l\}}\right)\right) .}\right.
\end{aligned}
$$

Since $(-1)^{|\Lambda|}+(-1)^{|\Lambda-\{l\}|}=0$ and $\left.g\right|_{G_{\Lambda}} \circ \varphi_{\Lambda}$ and $\left.g\right|_{G_{\Lambda-\{l\}}} \circ \varphi_{\Lambda-\{l\}}$ are ambient isotopic we have $v_{i}(g)=0$. Therefore we have $v_{i}(f)=0$. 
Now suppose that $C$ is an $i$-configuration on $G\left(e_{1}, \cdots, e_{m}\right)$ with $i \leq n$. Since $2 i \leq 2 n<m$ there is a pair of multiple edges $e_{l}$ and $d_{l}$ that have no points of $C$. Let $f_{C} \in \mathrm{SE}_{i}\left(G\left(e_{1}, \cdots, e_{m}\right)\right)$ be a realization of $C$ such that $e_{l}$ and $d_{l}$ are parallel in $f_{C}$. Then we have $v_{i}\left(f_{C}\right)=0$. Thus the condition (3) of Theorem A holds. Since there is an embedding $f \in \operatorname{SE}\left(G\left(e_{1}, \cdots, e_{m}\right)\right)$ with $v(f)=0$ we have the conclusion.

A $j$-cycle graph is a graph on $j$ vertices that is homeomorphic to a circle. A $j$-cycle of $G$ is a subgraph of $G$ that is a $j$-cycle graph. Let $\mathrm{C}_{j}(G) \subset \Omega(G)$ be the set of all $j$-cycles of $G$ and $\mathrm{C}(G)=\cup_{j=2}^{\infty} \mathrm{C}_{j}(G)$.

A graph $G$ is called adaptable if any set of embeddings $\left\{\phi_{\gamma} \in \operatorname{SE}(\gamma) \mid \gamma \in\right.$ $\mathrm{C}(G)\}$ is realizable up to ambient isotopy. In [5] Kinoshita showed that a graph on two vertices and some edges joining them is adaptable. Then in [24] Yamamoto showed that $K_{4}$ is adaptable. Then in [25] Yasuhara found a nice construction method of knots in a spatial graph using the fact that any knot is transformed into a trivial knot by delta unknotting operation introduced in [11]. For example the graph obtained from $K_{5}$ by deleting an edge is shown to be adaptable. On the other hand it is shown in [10] that no nonplanar graph is adaptable.

Let $C_{m}$ be an $m$-cycle graph and $e_{1}, \cdots, e_{m}$ the edges of $C_{m}$. Let $D_{m}=$ $C_{m}\left(e_{1}, \ldots, e_{m}\right)$.

The following is a corollary of Theorem 3.1 (1).

Corollary 3.2. The graph $D_{5}$ is not adaptable.

We note that the graph $D_{5}$ is the first planar graph which is shown to be non-adaptable. A graph $H$ is called a minor of a graph $G$ if $H$ is obtained from $G$ by a sequence of edge-contraction, edge-deletion and vertex-deletion. If $H$ is a minor of $G$ then there is a natural injection from $\mathrm{C}(H)$ into $\mathrm{C}(G)$. Therefore it follows that a minor of an adaptable graph is adaptable. In [22] the second author and Yasuhara showed that any proper minor of $D_{5}$ is adaptable. We also note that after our work some other planar graphs are shown to be non-adaptable in $[\mathbf{2 2}]$.

Proof. Let $v_{C_{5}}: \mathrm{SE}\left(C_{5}\right) \rightarrow Z$ be an invariant defined by $v_{C_{5}}(f)=a_{2}\left(f\left(C_{5}\right)\right)$ for each element $f \in \mathrm{SE}\left(C_{5}\right)$ where $a_{2}\left(f\left(C_{5}\right)\right)$ denotes the second coefficient of the Conway polynomial of the knot $f\left(C_{5}\right)$. Then $v_{C_{5}}$ is a Vassiliev type invariant of order at most 2. Since $2<\frac{5}{2}$ we have that $v=v\left(\left\{v_{\gamma}\right\}, \omega\right)$ : $\mathrm{SE}\left(D_{5}\right) \rightarrow Z$ is the zero map. On the other hand $a_{2}$ (trefoil knot) $=1$. Therefore a collection of knots, one trefoil knot by a 5-cycle and the others trivial, cannot be realized. This completes the proof.

Let $f \in \operatorname{SE}_{i}(G)$ and $c$ a self-crossing vertex of $f$ on an edge $e$ of $G$. Let $e^{\prime}$ be a subarc of $e$ whose end points are $f^{-1}(c)$. We say that $c$ is immediate if $f\left(e^{\prime}\right)$ contains no crossing vertices of $f$ except $c$. We say that $c$ is nugatory 
if $c$ is immediate and $f\left(e^{\prime}\right)$ bounds a disk in $R^{3}$ whose interior is disjoint from $f(G)$.

Let $f \in \mathrm{SE}_{i}(G)$ and $c$ a crossing vertex of $f$ of two adjacent edges $e$ and $d$ of $G$. Let $p$ be a common vertex of $e$ and $d$. Let $e^{\prime}$ and $d^{\prime}$ be subarcs of $e$ and $d$ respectively whose end points are $f^{-1}(c)$ and $p$. We say that $c$ is immediate if $f\left(e^{\prime} \cup d^{\prime}\right)$ contains no crossing vertices of $f$ except $c$. We say that $c$ is nugatory if $c$ is immediate and $f\left(e^{\prime} \cup d^{\prime}\right)$ bounds a disk in $R^{3}$ whose interior is disjoint from $f(G)$.

We note that if $c$ is a nugatory self-crossing vertex or a nugatory crossing vertex of adjacent edges then the positive resolution and the negative resolution of $f$ at $c$ are mutually ambient isotopic. Therefore if $f \in \mathrm{SE}_{i}(G)$ has a nugatory self-crossing vertex or a nugatory crossing vertex of adjacent edges then $v_{i}(f)=0$.

Proof of Theorem 3.1 (2). Let $C$ be an $i$-configuration on $G\left(e_{1}, \cdots, e_{m}\right)$ with $i \leq n$. Suppose that $C$ has a pair of points $p_{1}, p_{2}$ on an edge $e$ of $G\left(e_{1}, \cdots\right.$, $\left.e_{m}\right)$. If there are no other points of $C$ on $e$ between $p_{1}$ and $p_{2}$, then there is a realization $f_{C} \in \mathrm{SE}_{i}\left(G\left(e_{1}, \cdots, e_{m}\right)\right)$ of $C$ that has a nugatory self-crossing vertex. Therefore we have $v_{i}\left(f_{c}\right)=0$. If there are some other points of $C$ on $e$ then we have by the condition $i \leq n<(m+2) / 2$ that there is a pair of multiple edges $e_{l}$ and $d_{l}$ that have no points of $C$. Let $f_{C} \in \operatorname{SE}_{i}\left(G\left(e_{1}, \cdots, e_{m}\right)\right)$ be a realization of $C$ such that $e_{l}$ and $d_{l}$ are parallel in $f_{C}$. Then we have $v_{i}\left(f_{C}\right)=0$. Thus we have established the condition (3) of Theorem B.

Now the proof of Theorem 3.1 (3) is quite similar and we omit it.

Let $\Lambda$ be a subset of $\{1, \ldots, m\}$. Then we say that an $i$-singular embed$\operatorname{ding} f$ of $G\left(e_{1}, \ldots, e_{m}\right)$ is locally parallel with respect to $\Lambda$ if $f\left(\cup_{l \in \Lambda}\left(e_{l} \cup d_{l}\right)\right)$ contains no crossing vertices of $f$ and for each $l \in \Lambda e_{l}$ and $d_{l}$ are parallel in the restriction map $\left.f\right|_{\cup_{l \in \Lambda}}\left(e_{l} \cup d_{l}\right)$.

Similarly we say that an $i$-singular embedding $f$ of $G\left(e_{1}, \ldots, e_{m}\right)$ is locally parallel up to edge-homotopy (resp. vertex-homotopy) with respect to $\Lambda$ if $f\left(\cup_{l \in \Lambda}\left(e_{l} \cup d_{l}\right)\right)$ contains no crossing vertices of $f$ and the restriction map $f \mid \cup_{l \in \Lambda}\left(e_{l} \cup d_{l}\right)$ is edge-homotopic (resp. vertex-homotopic) to an element $h$ of $\mathrm{SE}\left(\cup_{l \in \Lambda}\left(e_{l} \cup d_{l}\right)\right)$ such that for each $l \in \Lambda e_{l}$ and $d_{l}$ are parallel in $h$.

The following three theorems are applications of Theorem D.

Theorem 3.3. Let $\Lambda$ be a subset of $\{1, \cdots, m\}$ with $|\Lambda|=k$. If $n \leq k-1$ and $f \in \mathrm{SE}\left(G\left(e_{1}, \cdots, e_{m}\right)\right)$ is locally parallel with respect to $\Lambda$ then $v(f)=$ $v\left(\left\{v_{\gamma}\right\}, \omega\right)(f)=0$.

Theorem 3.4. Let $k$ be a natural number with $k \leq m-1$. Let $f$ and $g$ be elements of $\operatorname{SE}\left(G\left(e_{1}, \cdots, e_{m}\right)\right)$ each of which is locally parallel up to edgehomotopy with respect to any $\Lambda \subset\{1, \cdots, m\}$ with $|\Lambda|=k$. Suppose that $n \leq k$ and $f$ and $g$ are edge-homotopic. Then $v(f)=v\left(\left\{v_{\gamma}\right\}, \omega\right)(f)=v(g)=$ $v\left(\left\{v_{\gamma}\right\}, \omega\right)(g)$. 
Theorem 3.5. Let $k$ be a natural number with $k \leq m-2$. Let $f$ and $g$ be elements of $\operatorname{SE}\left(G\left(e_{1}, \cdots, e_{m}\right)\right)$ each of which is locally parallel up to vertex-homotopy with respect to any $\Lambda \subset\{1, \cdots, m\}$ with $|\Lambda|=k$. Suppose that $n<\frac{2}{3}(k+2)$ and $f$ and $g$ are vertex-homotopic. Then $v(f)=$ $v\left(\left\{v_{\gamma}\right\}, \omega\right)(f)=v(g)=v\left(\left\{v_{\gamma}\right\}, \omega\right)(g)$.

Note that in many situations these theorems actually present sharper results on the order $n$ of $v$ than that in Theorem 3.1. We also note that an edge-homotopy invariant for $D_{3}$ defined in [18] is an example of Theorem 3.4 where $k=n=2, \mathcal{R}=Z / 2 Z$ and $v_{\gamma}$ is the Arf invariant of knots. In general it follows from Theorem 3.4 that the spatial graphs in Figure 1.2 are not edge-homotopic to plane graphs. See Example 3.6.

Proof of Theorem 3.3. For $1 \leq i \leq k$ let $\mathrm{T}_{i}\left(G\left(e_{1}, \cdots, e_{m}\right)\right)$ be the $i$-singular embeddings of $G\left(e_{1}, \cdots, e_{m}\right)$ that is locally parallel with respect to some $\Delta \subset \Lambda$ with $|\Delta|=k-i$. Let $g \in \mathrm{T}_{i}\left(G\left(e_{1}, \cdots, e_{m}\right)\right)$. Suppose that $g$ is locally parallel with respect to $\Delta$ and $l \in \Delta$. Let $D$ be a disk that bounds $g\left(e_{l} \cup d_{l}\right)$. If the interior of $D$ is disjoint from $g\left(G\left(e_{1}, \cdots, e_{m}\right)\right)$ then we have $v_{i}(g)=0$ as in the proof of Theorem 3.1 (1). If not then we remove the intersection by a sequence of crossing changes. Let $h \in$ $\mathrm{T}_{i}\left(G\left(e_{1}, \cdots, e_{m}\right)\right)$ be the result of the crossing changes. Then we have that $e_{l}$ and $d_{l}$ are parallel in $h$ and $v_{i}(h)=0$. Moreover it is clear that the $(i+1)$-singular embeddings corresponding to the crossing changes are contained in $\mathrm{T}_{i+1}\left(G\left(e_{1}, \cdots, e_{m}\right)\right)$. Therefore we have by Theorem $\mathrm{D}$ that $v_{1}\left(\mathrm{~T}_{1}\left(G\left(e_{1}, \cdots, e_{m}\right)\right)\right)=0$. Suppose that $f \in \operatorname{SE}\left(G\left(e_{1}, \cdots, e_{m}\right)\right)$ is locally parallel with respect to $\Lambda$. Then similarly there is a sequence of crossing changes from $f$ to some $u \in \mathrm{T}\left(G\left(e_{1}, \cdots, e_{m}\right)\right)$ with $v(u)=0$ whose corresponding 1-singular embeddings are contained in $\mathrm{T}_{1}\left(G\left(e_{1}, \cdots, e_{m}\right)\right)$. Therefore we have $v(f)-v(u)=0$ and $v(f)=0$.

Proof of Theorem 3.4. It is sufficient to show that the values of $v_{1}$ of the 1-singular embeddings corresponding to the edge-homotopy from $f$ to $g$ are zero. For $1 \leq i \leq k$ let $\mathrm{T}_{i}\left(G\left(e_{1}, \cdots, e_{m}\right)\right)$ be the $i$-singular embeddings of $G\left(e_{1}, \cdots, e_{m}\right)$ each of which is locally parallel up to edge-homotopy with respect to some $\Delta$ with $|\Delta|=k-i+1$. Let $h \in \mathrm{T}_{i}\left(G\left(e_{1}, \cdots, e_{m}\right)\right)$. Suppose that $h$ is locally parallel up to edge-homotopy with respect to $\Delta$ and $l \in \Delta$. Then we choose a sequence of self-crossing changes from $h$ to an element $u \in \mathrm{T}_{i}\left(G\left(e_{1}, \cdots, e_{m}\right)\right)$ that is locally parallel with respect to $\Delta$ and then choose a sequence of crossing changes from $u$ to an element $w \in$ $\mathrm{T}_{i}\left(G\left(e_{1}, \cdots, e_{m}\right)\right)$ in which $e_{l}$ and $d_{l}$ are parallel. Then we have that $v_{i}(w)=$ 0 and all the corresponding $(i+1)$-singular embeddings are contained in $\mathrm{T}_{i+1}\left(G\left(e_{1}, \cdots, e_{m}\right)\right)$. Then we have the result by Theorem $\mathrm{D}$.

Proof of Theorem 3.5. It is sufficient to show that the values of $v_{1}$ of the 1singular embeddings corresponding to the vertex-homotopy from $f$ to $g$ are 
zero. For $1 \leq i \leq n$ let $\mathrm{T}_{i}\left(G\left(e_{1}, \cdots, e_{m}\right)\right)$ be the $i$-singular embeddings of $G\left(e_{1}, \cdots, e_{m}\right)$ each of which is locally parallel up to vertex-homotopy with respect to some $\Delta$ with $|\Delta|=s \geq 0$ and has at least $t \geq 0$ immediate crossing vertices of adjacent edges for some $s$ and $t$ with $\frac{2}{3} s+\frac{1}{3} t>n-i$. Let $h \in \mathrm{T}_{i}\left(G\left(e_{1}, \cdots, e_{m}\right)\right)$.

Case 1. $h$ is locally parallel up to vertex-homotopy with respect to some $\Delta \neq \emptyset$.

Let $|\Delta|=s$. Then $h$ has at least $t$ immediate crossing vertices of adjacent edges and $\frac{2}{3} s+\frac{1}{3} t>n-i$. We choose a sequence of crossing changes of adjacent edges from $h$ to an element $u$ in $\mathrm{T}_{i}\left(G\left(e_{1}, \cdots, e_{m}\right)\right)$ that is locally parallel with respect to $\Delta$. We note that each of the corresponding $(i+1)$ singular embeddings is locally parallel with respect to some (possibly empty) subset $\Delta^{\prime}$ of $\Delta$ with $\left|\Delta^{\prime}\right| \geq s-2$ and has at least $t+1$ immediate crossing vertices of adjacent edges. Therefore it is contained in $\mathrm{T}_{i+1}\left(G\left(e_{1}, \cdots, e_{m}\right)\right)$. Next we choose a sequence of crossing changes from $u$ to an element $w$ in $\mathrm{T}_{i}\left(G\left(e_{1}, \cdots, e_{m}\right)\right)$ such that $e_{l}$ and $d_{l}$ are parallel in $w$ for some $l \in \Delta$. Then we have $v_{i}(w)=0$. We note that each of the $(i+1)$-singular embeddings corresponding to the crossing changes from $h$ to $w$ is locally parallel with respect to $\Delta-\{l\}$ and has at least $t-1$ immediate crossing vertices of adjacent edges. Therefore they are contained in $\mathrm{T}_{i+1}\left(G\left(e_{1}, \cdots, e_{m}\right)\right)$.

Case 2. $h$ is not locally parallel up to vertex-homotopy with respect to any $\Delta \neq \emptyset$.

Then we have that $h$ has at least $3(n-i)+1$ immediate crossing vertices of adjacent edges. Then we choose a sequence of crossing changes from $h$ to $u$ that has a nugatory crossing vertex of adjacent edges. Then $v_{i}(u)=0$ and each of the corresponding $(i+1)$-singular embeddings still has at least $3(n-i)+1-2>3(n-(i+1))+1$ immediate crossing vertices of adjacent edges. Therefore they are contained in $\mathrm{T}_{i+1}\left(G\left(e_{1}, \cdots, e_{m}\right)\right)$.

Thus by Theorem $\mathrm{D}$ we have the result.

Example 3.6. Let $J_{m}$ be the knot illustrated in Figure 3.1.

By a calculation we have that the Jones polynomial of $J_{m}$

$$
V_{J_{m}}(t)=1+\left(1+t^{-2}\right)^{m-3}\left(t^{2}+t+1\right)\left(1-t^{-1}\right)^{m-1} .
$$

Then by a calculation we have that the $(m-1)$-th derivative of $V_{J_{m}}(t)$ evaluated at $1 V_{J_{m}}^{(m-1)}(1)=3 \cdot(m-1) ! \cdot 2^{m-3} \neq 0$. It is well known that $V_{J}^{(m-1)}(1)$ is an order $m-1$ Vassiliev type invariant of a knot $J$. We note that each of the spatial graphs in Figure 1.2 contains only one nontrivial knot $J_{m}$. Therefore by Theorem 3.4 we have that the spatial graphs in Figure 1.2 are not edge-homotopic to plane graphs. We remark that they are vertex-homotopic to plane graphs. 


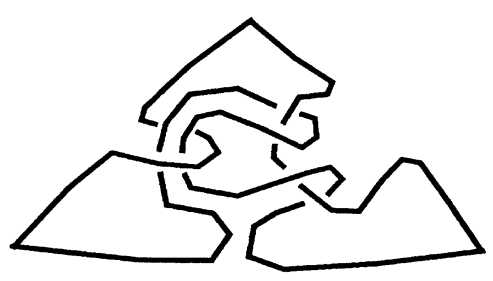

$J_{3}$

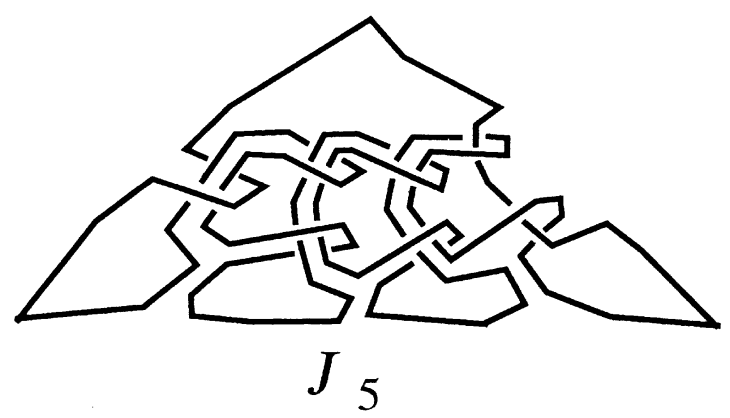

Figure 3.1.

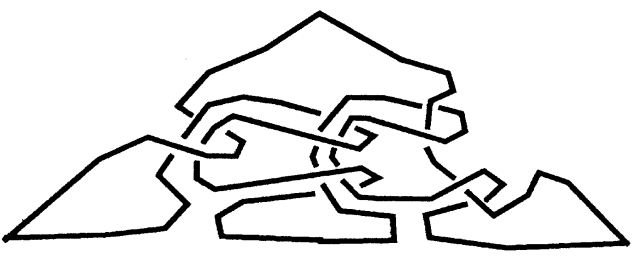

$J_{4}$

\section{References}

[1] D. Bar-Natan, On the Vassiliev invariants, Topology, 34 (1995), 423-472, Zbl 898.57001.

[2] J. Birman and X.-S. Lin, Knot polynomials and Vassiliev's invariants, Invent. Math., 111 (1993), 225-270, MR 94d:57010, Zbl 812.57011.

[3] J.H. Conway and C.McA. Gordon, Knots and links in spatial graphs, J. Graph Thory, 7 (1983), 445-453, MR 85d:57002, Zbl 524.05028.

[4] M. Gusarov, A new form of the Conway-Jones polynomial of oriented links, Nauchn. Sem. Len. Otdel. Mat. Inst. Steklov, 193 (1991), 4-9, MR 93b:57007, Zbl 747.57005.

[5] S. Kinoshita, On $\theta_{n}$-curves in $R^{3}$ and their constituent knots, in 'Topology and Computer Science' edited by S. Suzuki, Kinokuniya, (1987), 211-216, MR 92h:57010.

[6] _ On spatial bipartite $K_{m, n}$ 's and their constituent $K_{2, n}$ 's, Kobe J. Math., 8 (1991), 41-46, MR 92h:57004, Zbl 765.57002.

[7] T. Kohara and S. Suzuki, Some remarks on knots and links in spatial graphs, Knots 90, ed. A. Kawauchi, Walter de Gruyter, Berlin-New York, (1992), 435-445. MR 93i:57004, Zbl 771.57002.

[8] X.-S. Lin, Finite type link invariants of 3-manifolds, Topology, 33 (1994), 45-71, MR 94m:57020, Zbl 816.57013.

[9] J. Milnor, Link groups, Ann. Math., 59 (1954), 177-195, MR 17,70e, Zbl 055.16901.

[10] T. Motohashi and K. Taniyama, Delta unknotting operation and vertex homotopy of graphs in $R^{3}$, Proceedings of Knots 96 (S. Suzuki ed.), World Sci. Publ. Co., (1997), 185-200, MR 99i:57021. 
[11] H. Murakami and Y. Nakanishi, On a certain move generating link-homology, Math. Ann., 284 (1989), 75-89, MR 90f:57007, Zbl 646.57005.

[12] Y. Ohyama, Vassiliev invariants and similarity of knots, Proc. Amer. Math. Soc., 123 (1995), 287-291, MR 95f:57018, Zbl 847.57012.

[13] _ Twisting of two strings and Vassiliev invariants, Topology Appl., 75 (1997), 201-215, MR 98a:57013, Zbl 876.57016.

[14] T. Otsuki, Knots and links in certain spatial complete graphs, J. Combin. Theory Ser. B., 68 (1996), 23-35, MR 97g:57013, Zbl 858.05038.

[15] N. Robertson, P. Seymour and R. Thomas, Linkless embeddings of graphs in 3-space, Bull. Amer. Math. Soc., 28 (1993), 84-89, MR 93f:57006, Zbl 769.05034.

[16] H. Sachs, On spatial analogue of Kuratowski's theorem on planar graphs, Lecture Notes in Math., 1018, Springer-Verlag, Berlin-Heidelberg, (1983), 230-241, MR 85b:05077, Zbl 525.05024.

[17] T. Stanford, Finite-type invariants of knots, links and graphs, Topology, 35 (1996), 1027-1050, MR 97i:57009, Zbl 863.57005.

[18] K. Taniyama, Cobordism, homotopy and homology of graphs in $R^{3}$, Topology, 33 (1994), 509-523, MR 95h:57002, Zbl 823.57006.

[19] Link homotopy invariants of graphs in $R^{3}$, Rev. Mat. Univ. Complut. Madrid, 7 (1994), 129-144, MR 95f:57023, Zbl 818.57005.

[20] Knotted subgraphs in a spatial graph, Kobe J. Math., 14 (1997), 207-212, MR 98m:57006, Zbl 901.05037.

[21]__ Higher dimensional links in a simplicial complex embedded in a sphere, Pacific J. Math., 194(2) (2000), 465-467, CMP 1760794.

[22] K. Taniyama and A. Yasuhara, Realization of knots and links in a spatial graph, preprint.

[23] V. Vassiliev, Cohomology of knot spaces, Theory of singurarities and its applications (V. Arnold ed.), Advances in Soviet Mathematics, 1, AMS Providence, RI, 1990, MR 92a:57016, Zbl 727.57008.

[24] M. Yamamoto, Knots in spatial embeddings of the complete graph on four vertices, Topology Appl., 36 (1990), 291-298, MR 91m:57007, Zbl 721.57005.

[25] A. Yasuhara, Delta-unknotting operation and adaptability of certain graphs, Proceedings of Knots 96 (S. Suzuki ed.), World Sci. Publ. Co., (1997), 115-121, MR 99j:57004.

\author{
Department of Intelligence and Computer Science \\ Nagoya Institute of TeChNology \\ GOKISO, SHOWA-KU \\ NAGOYA, 466-8555 \\ JAPAN \\ E-mail address: ohyama@math.kyy.nitech.ac.jp \\ Department of Mathematics \\ TOKYO WOMAn's Christian University \\ Zempukuji 2-6-1, Suginamiku \\ TOKYO 167-8585 \\ JAPAN \\ E-mail address: taniyama@twcu.ac.jp
}


